\title{
Internal Numerical Simulation of a Swirl Simplex Atomizer to Predict Atomization Outputs
}

\author{
D. Ferrando*1, M. Belmar-Gil ${ }^{2}$, L. Palanti ${ }^{3}$, M. Carreres ${ }^{2}$, D. Cervelló-Sanz² ${ }^{2}$ F.X. Demoulin ${ }^{1}$, \\ B. Renou ${ }^{1}$, B. Duret ${ }^{1}$, J. Reveillon ${ }^{1}$ \\ ${ }^{1}$ CNRS CORIA UMR 6614, University of Rouen Normandie, France \\ ${ }^{2}$ CMT-Motores Térmicos, Universitat Politècnica de València, Spain \\ ${ }^{3}$ Department of Industrial Engineering (DIEF), University of Florence, Italy \\ ${ }^{*}$ Corresponding author email: diego.ferrando@coria.fr
}

\begin{abstract}
Numerical simulation of injectors could help improving their designs. In particular, a two-phase flow simulation of a swirl simplex atomizer allows computing the main characteristic parameters such as the spray cone angle, the fuel sheet thickness and the air core diameter. This work describes an attempt to go a step further to characterize atomization and spray characteristics with the ultimate goal to predict the spray distribution in terms of size and velocity.

A commercial injector has been used in the CORIA Rouen Spray Burner (CRSB) set up to study turbulent spray combustion. This experiment has produced a well-documented database for the last two TCS workshops (see TCS 6 and 7 at http://www.tcs-workshop.org/). Even though spray measurements are used to define the fuel injection in combustion numerical simulations, the spray injection characteristics remain the main cause of uncertainties. The present work aims at answering this question: Is it possible to complete our knowledge of spray injection by numerical simulation of the full atomization process?

The first challenge is to measure the inner geometry. Standards techniques such as X-ray tomography and microscopy have been applied. In order to accurately reproduce the internal throat and swirl chamber, additional measurements have been performed by silicon molding, leading to the definition of a nominal geometry that can be used for further CFD simulation. The second challenge is to produce a mesh regular enough to be compatible with the interface capturing method. Several mesh strategies have been tested starting from the swirl chamber with very thin mesh layers to capture the liquid film at the injector wall and to describe the external liquid sheet. Then, a third challenge concerns the numerical simulation itself, since it must handle the multi-scale nature of such flows until the formation of the spray. A nonreactive condition has been tested with the interFoam solver (OpenFOAM library) in a Large Eddy Simulation (LES) framework.

Eventually, the last challenge is to build an analysis to connect the numerical simulation that is limited to the close vicinity of the injector and the experimental measurements that are rejected farther downstream on the dispersed spray. This last step is based on the analysis of the surface curvature distribution that is described with more detail on another presentation at this ICLASS conference.
\end{abstract}

\section{Keywords}

swirl-simplex-atomizer, two-phase flow, numerical simulation, atomization

\section{Introduction}

In pressure-swirl (simplex) atomizers, fuel is fed to an internal chamber through several tangential ports. The chamber outlet constitutes the atomizer orifice. Hence, the swirling motion in the chamber generates an air core so that the fuel is atomized to the exterior as a hollow cone. The swirling motion allows the liquid sheet to spread out achieving wider cone angles than those obtained with plain-orifice atomizers [1]. The applications of these atomizers are 
diverse, including agricultural spraying processes, paint spraying, spray drying of wet solids, food processing and several combustion applications [2, 3].

Focusing on combustion applications, these include internal combustion engines, gas turbine engines and others [4], where the spray characteristics are of key relevance to the engine performance, fuel consumption and generation of emissions. While fossil fuels are being progressively replaced in land transportation, aircraft engines will still rely on their combustion for some decades. Last-generation lean-burn engines such as the GE-TAPS start making use of airblast atomizers together with simplex atomizers, the latter generating the pilot flame for engine startup and low-power operations [5].

Due to its importance in the industry, research studies on the atomization features of this kind of atomizer are varied. Experiments include high-speed imaging and the application of optical techniques such as Phase Doppler Anemometry (PDA) [6, 7], Laser Doppler Anemometry (LDA) [8] or Mie-scattering [9]. These works reveal that the primary breakup takes place by the growth of disturbances on the liquid sheet that are generated by the external surrounding air as soon as the fuel leaves the atomizer. Nevertheless, quantitative studies are limited to the secondary atomization regime, as observation of ligament and droplet formation in the near-nozzle region is precluded by limitations of the optical techniques and experimental arrangements. These limitations can be overcome through computational techniques.

Many numerical studies are based on the transport equation of the liquid volume fraction method to treat the two-phase flow in a Eulerian framework. These approaches are often referred as Volume of Fluid (VOF) methods even if this appellation hides a wide range of treatment of the liquid volume flux. Turbulence modelling approaches include laminar assumption $[10,11]$, RANS [12] and LES [13, 14]. The computational domain generally includes the internal and the external flow, but none of these works seems to reproduce the instabilities in the liquid sheet experimentally reported to produce breakup, thus predicting different atomization mechanisms. Except for Laurila et al. [14], whose atomizer configuration differs from those of combustion applications, only works making use of costly DNS through VOF $[15,16]$ or Level Set [17] are able to capture the film instabilities triggering atomization.

The main objective of the present work is then to present a methodology that allows studying the primary atomization in pressure-swirl (simplex) atomizers. Three main stages are here reported to this end: on the measurement of the geometry, on the production of the mesh and on the development of a numerical strategy to handle the multi-scale nature of the problem.

The particular atomizer under study belongs to the CORIA Rouen Spray Burner (CRSB) setup that has been used to create a well-documented database of relevance for gas turbine applications and used for the two last TCS (Turbulent Combustion of Sprays) workshops [18]. Thus, some of the features of the generated spray have been experimentally investigated in the secondary breakup region $[19,20]$. The present investigation will then help filling the gap between this region and the atomizer outlet, thus allowing to generate realistic boundary conditions for standard Eulerian-Lagrangian simulations of the full burner geometry in a wide range of operating conditions.

\section{Experimental characterization of the geometry}

The simplex atomizer used in the CRSB burner is a Danfoss commercial nozzle. Its three main pieces shape the three fuel inlet tangential ports, the swirl chamber and the orifice duct, as shown in Figure $1(b)$. The geometrical details of the interior of the atomizer have been experimentally obtained via silicone molding of the outer piece, labelled as (1) in Figure 1(b). This technique was first applied to determine the internal geometry of Diesel nozzles by Macian et al. [21]. Metrology on the obtained molds and atomizer pieces is performed through a combination of three visualization techniques (see Figure 1), namely optical microscope visualization, Scanning Electron Microscope (SEM) visualization and Computed Tomography (CT) scan.

Please note that a gold coating (in the order of $\mathrm{nm}$ ) must be applied to the molds before visual- 


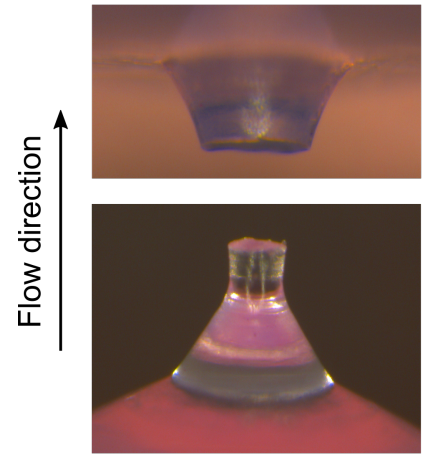

(a)

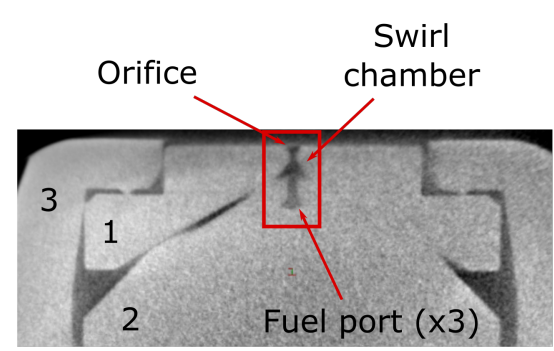

(b)

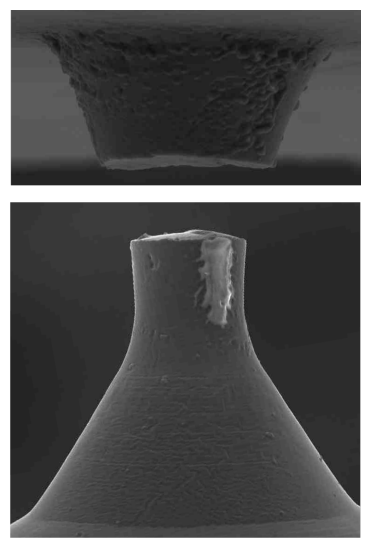

(c)

Figure 1. Sample pictures of the CRSB atomizer: (a) optical microscope visualization of the silicone molds, (b) CT scan of the atomizer assembly, (c) SEM visualization of the silicone molds.

izing them in the SEM so that they become conductor. The accuracy of the methodology has been reported to be around $2 \%$ in the determination of diesel injector nozzle outlet diameters [22].

As a result from the experimental metrology, the internal geometry of the atomizer is reconstructed as sketched in Figure 2. The main dimensions obtained are summarized in Table 1.

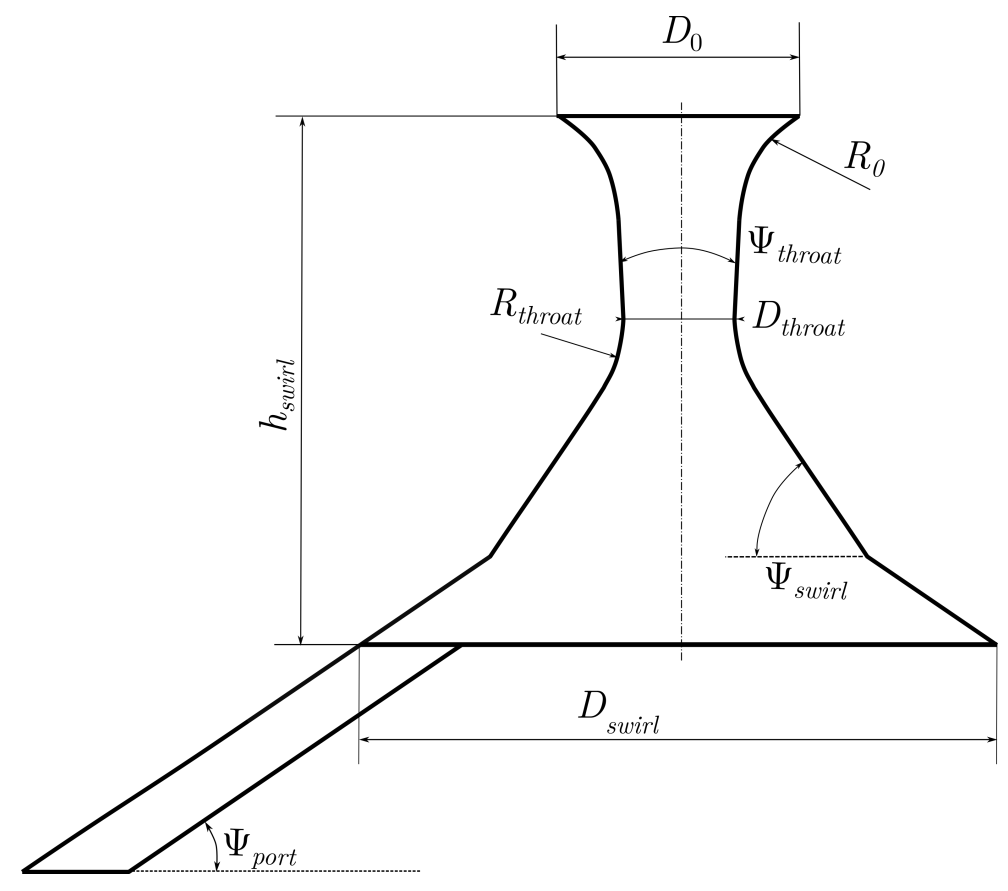

Figure 2. Sketch of the CRSB atomizer reconstructed internal geometry.

\section{Numerical methodology}

The numerical simulation is based on the well known OpenFoam library that already contains many features needed for liquid-gas flows simulation on complex geometries, with a built-in parallel computing ability to handle intensive simulation. Two important flow characteristics have to be considered: turbulence and the liquid-gas interface. Regarding turbulence, a LES approach has been used with the Smagorinsky model [23] and the WALE model [24]. The interface is implicitly captured through the liquid volume fraction $(\alpha)$ equation. Thus, the interface is localized at the transition between the gas $(\alpha=0)$ and the liquid $(\alpha=1)$. To keep an 
Table 1. Summary of the CRSB atomizer internal dimensions.

\begin{tabular}{c|cccccc}
\hline & $D_{0}$ & $D_{\text {throat }}$ & $D_{\text {swirl }}$ & $h_{\text {swirl }}$ & $R_{0}$ & $R_{t}$ \\
\hline Value $[\mu \mathrm{m}]$ & 340 & 165 & 780 & 620 & 310 & 190 \\
\hline & & $\Psi_{\text {throat }}$ & & $\Psi_{\text {swirl }}$ & & $\Psi_{\text {port }}$ \\
\hline Value $\left[{ }^{\circ}\right]$ & & 6 & & 60 & & 30
\end{tabular}

accurate description of the interface the transition should remain sharp with a size of the order of few $\Delta x$. On regular VOF approaches the interface position is reconstructed [26][27] and the liquid volume flux are based on the geometrical advection of this surface. This geometrical approach brings difficulties for the general polygons used in OpenFoam as numerical cells used to handle complex geometries. Thus, liquid advection is based on standard numerical schemes bringing numerical diffusion that potentially enlarges the transition zone. To counter this effect a sharpening term is introduced [28][29].

The numerical simulation has to deal with a wide length scale range. Hence, the mesh resolution needs to be fine enough at particular locations to accurately capture the smallest length scales. Starting in the internal zone of the injector, the mesh resolution has been refined in the normal direction to the wall in order to compute the boundary layer. The liquid film thickness inside the nozzle also needs to be accurately meshed. On the other hand, downstream of the nozzle, the liquid sheet is developed, becoming thinner and thinner, requiring additional mesh resolution. Finally, once the liquid sheet breaks up, the droplets are atomized. The mesh resolution has to be fine enough to capture the smallest droplets. As a conclusion, the computational meshing process is not straightforward and, thus, a workflow to handle the simulation has been proposed. The workflow of the simulations is shown in Figure 3.

First, the inner flow has to be modelled. A full 3D simulation of the atomizer internal flow (swirl chamber and the three fuel ports) and $2 \mathrm{~mm}$ of the external region is carried out to this end. Data on the velocity coordinates and the liquid volume fraction are sampled at an orifice crosssection slightly upstream of the atomizer outlet. As the second step, these data are then fed to a more refined sector simulation $\left(25^{\circ}\right)$ of the external domain where the spray is captured. These simulations have been run in a preliminary manner with the ultimate goal to perform a single full domain simulation which could handle the multi-scale nature of the simplex atomizer. The operating condition tested has been chosen from the guidelines of TCS7 [18], from where some experimental data and reference images taken in the CRSB are available. The main parameters used to define the boundary conditions of the simulations are shown in Table 2.

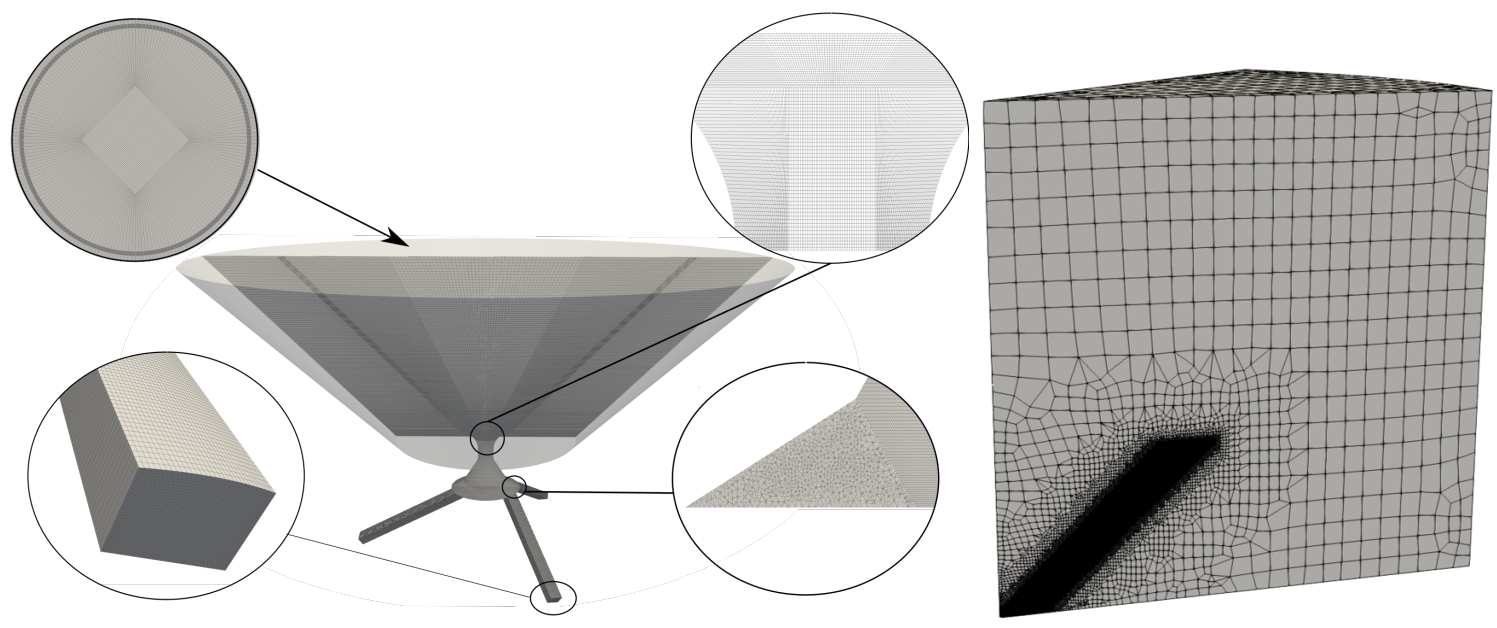

Figure 3. Simulation workflow: internal flow simulation (left) and external flow simulation (right). 
Table 2. Summary of the operating condition tested.

\begin{tabular}{c|cccc}
\hline & Fuel & $\dot{m}_{f}[\mathrm{~g} / \mathrm{s}]$ & $T$ & $p[\mathrm{MPa}]$ \\
\hline Value & n-Heptane & 0.28 & 298 & 0.1
\end{tabular}

\section{Internal flow simulation}

As depicted in Figure 3, the mesh for the internal flow simulation is hybrid: a structured mesh is adopted at the fuel ports and for most of the atomizer interior. A transition region between the fuel ports and the structured region of the swirl chamber is filled with polyhedra to overcome the limitations posed by the asymmetry of the channels. Special refinement is adopted near the walls, both to keep the $y^{+}$below 5 at the walls anywhere in the domain (no wall functions are then used) and to represent the liquid film inside the atomizer with at least 10 cells in the radial direction. A mesh sensitivity study (not shown for the sake of brevity) has been carried out considering the purpose of this simulation is to feed the sector simulation. A compromise between accuracy and cost provided a mesh with a total of $13 \mathrm{M}$ cells.

The WALE (Wall-Adapting Local Eddy-viscosity) sub-grid scale model introduced by Nicoud and Ducros [24] has been used as it theoretically replicates the proper near-wall scaling for the subgrid scale eddy-viscosity at the wall. Please note that the situation is even more complex since it concerns a two-phase flow boundary layer for which no model has been proposed up to now. As boundary conditions, the fuel mass flow rate from Table 2 prescribes the velocity at the three inlet ports. Atmospheric pressure is set at the outlet. A no-slip condition has been used at the physical walls, whereas a zero gradient condition is used at the outer external boundaries. Concerning the numerical setup, a PIMPLE algorithm is employed. $2^{\text {nd }}$ order accurate schemes are chosen both in space and time. Variable time-stepping is used, the maximum CFL number being limited to 0.4 . This results in time steps in the order of $2.5 \times 10^{-9} \mathrm{~s}$. The simulation is run for a physical time of $0.4 \mathrm{~ms}$, with a cost of $32,000 \mathrm{CPU} \cdot \mathrm{h}$. Data for the external flow simulation were sampled every $5 \times 10^{-8} \mathrm{~s}$ for $0.1 \mathrm{~ms}$.

The main flow features obtained at the atomizer mid-plane are shown in Figure 4. From the liquid volume fraction representation, a spray angle of $82^{\circ}$ is recovered in good agreement with the manufacturer's nominal opening angle $\left(80^{\circ}\right)$. A liquid film thickness of $15 \mu \mathrm{m}$ is obtained at the outlet, in agreement with Lefebvre's correlation [1]. However, the diffuse behavior of the interface at the external part highlights the need for additional refinement. Despite the air core fluctuating motion at the bottom of the chamber, the instantaneous and averaged fields downstream of the throat resemble each other. The important radial velocities lead to a computed swirl number $S_{w} \approx 1.5$ at the chamber inlet plane, $S_{w} \approx 2.5$ in the chamber and $S_{w} \approx 0.5$ at the orifice outlet. The axial velocity contour shows a shear layer around the liquid-gas interface, with an important acceleration of the liquid film from the throat to the outlet and an accused air entrainment at the orifice axis.

\section{External flow: sector simulation}

The instability of the liquid sheet is what triggers the primary break-up, thus, it must be captured in the numerical simulation. This physical phenomena happens due to the combination of the streamwise shear and the centrifugal instability due to the azimuthal motion within the liquid sheet[25]. Capturing the liquid sheet instabilities requires a well refined computational mesh and an extended domain in axial and radial directions as reported in [14] [16].

In order to define the domain length and the mesh resolution, several 2D asymmetric simulations have been carried out. The aim of this work is to be able to catch these instabilities and the atomization process in a sector 3D simulation and later on in a single simulation of the complete injector.

Three different mesh resolutions have been used to study the mesh sensitivity. The most refined cell sizes located around the liquid sheet are $2 \mu \mathrm{m}, 1 \mu \mathrm{m}$ and $0.5 \mu \mathrm{m}$. The domain begins 

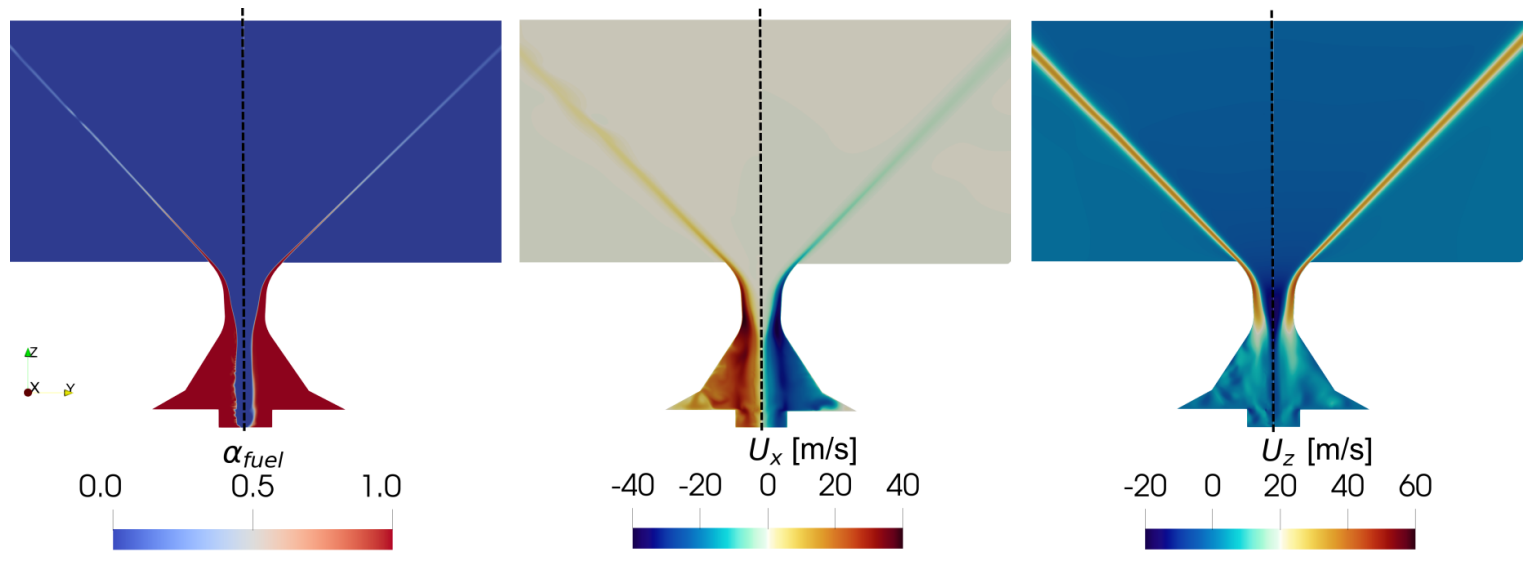

Figure 4. Mid-plane contours of liquid volume fraction (left), radial velocity (center) and axial velocity (right). In each case the left-hand side represents instantaneous data and the right-hand side shows averaged data.

at the throat of the atomizer (where the sampling surface has been recorded) and is extended up to 15 times the nozzle diameter in both axial and radial directions.

As it has been reported for the internal flow simulation, the WALE sub-grid scale model has been retained. As boundary conditions, Dirichlet time-varying boundary conditions have been used both for the velocity and the liquid volume fraction. This boundary condition has been mapped from the sampling of the internal simulation. Atmospheric pressure is set at the outlet and no-slip boundary condition has been set at the wall.

The liquid sheet destabilisation is captured only for mesh resolution with less than $1 \mu \mathrm{m}$. On the other hand, with a cell size of $2 \mu \mathrm{m}$ the liquid sheet remains straight up to a point where the break-up occurs by lack of resolution (Figure 5).
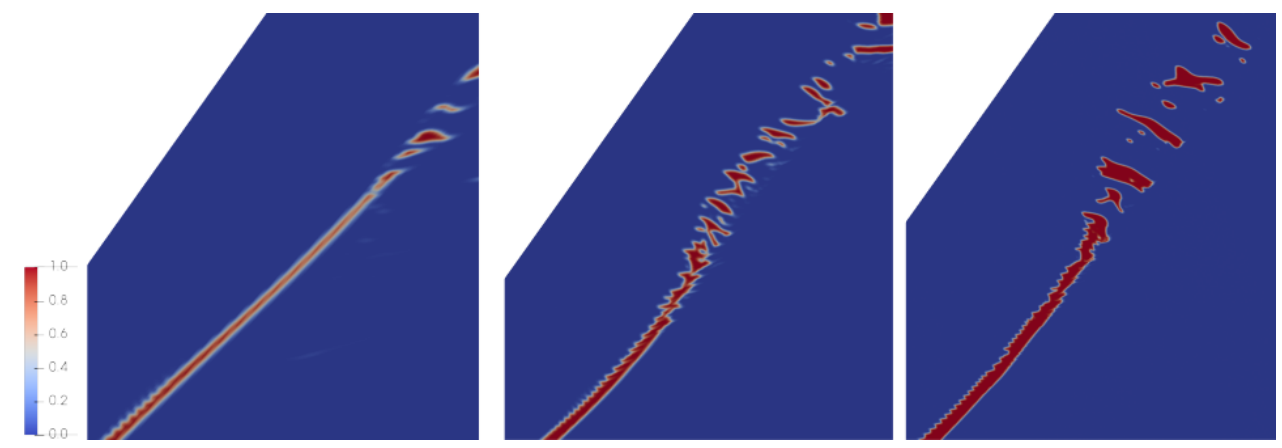

Figure 5. Liquid sheet instabilities on different 2D asymmetric resolution meshes: $2 \mu \mathrm{m}$ (left), $1 \mu \mathrm{m}$ (center), $0.5 \mu \mathrm{m}$ (right)

Further work is going on following the same methodology but with a sector domain using a periodic boundary condition. As Figure 6 shows (the sector simulation has been replicated and rotated to fulfill $360^{\circ}$ ), the liquid sheet seems more stable than in the $2 \mathrm{D}$ test cases despite the fine mesh resolution that has been preserved. However, the liquid-gas interface defined as the iso-contour of the liquid volume fraction $(\alpha=0.5)$ looks qualitatively in agreement with the experimental pictures. The angle of the spray is correctly reproduced and the size of the liquid droplet seems of the same order of magnitude. However, the liquid sheet instability on the experimental side seems to produce a more wavy behaviour than in the simulation. We are presently using the sector simulation extensively in order to understand what could trigger this wavy behaviour on the liquid sheet. It is also possible that the interpolation induced by the connection between the internal and external sector simulations damped the transmission of the internal fluctuations. This preparatory simulation has set the characteristics of the full single simulation (around $200 \mathrm{M}$ elements and 500,000 CPU.h) that will be tested an presented at the 
ICLASS meeting.
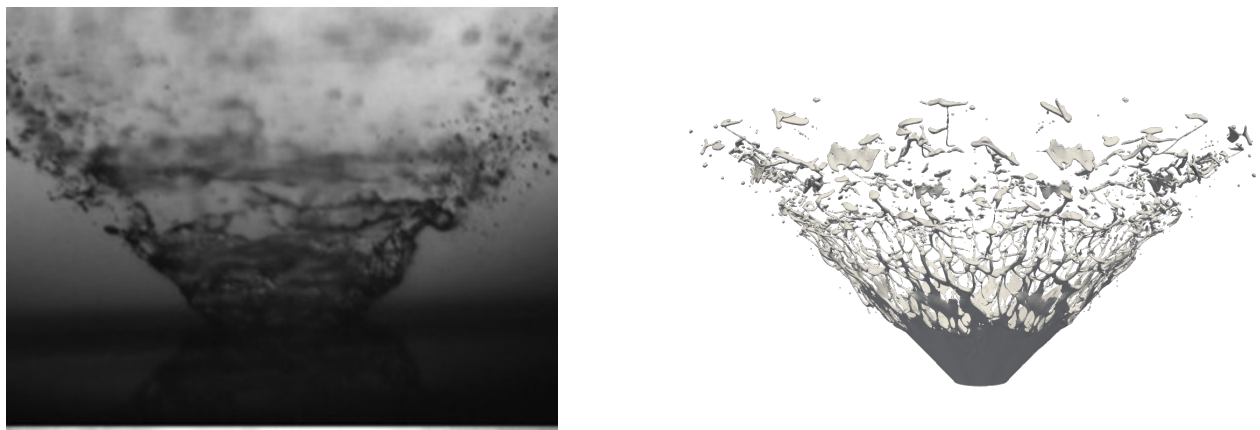

Figure 6. Comparison among experiments and sector simulation

\section{Conclusions}

In this paper, we have presented a methodology to model a commercial simplex-injector. The measurements of the internal geometry of the injector have been obtained by metrology techniques such as optical microscope visualization, SEM visualization and CT scan. From this internal geometry, several computational meshes have been designed to fulfill the mesh refinement requirement necessary to handle the small scale features of this atomization process. A hybrid mesh has been used to handle the non-asymmetric internal geometry prescribed by the fuel inlet ports. In order to optimize the computational cost, a methodology has been proposed to be able to capture the liquid sheet and the droplets with an interface capturing method based on the OpenFoam library. The process has been divided in an internal simulation where the air core is developed reaching the bottom wall of the injector. The angle of the spray is well captured and in a good agreement with the experimental measurements. The second part of this methodology is to simulate the primary break-up itself, which takes place outside of the injector. Several 2D asymmetric simulations have been carried out to determine the minimal mesh resolution required to catch the liquid sheet instabilities. A first work based on the 3D sector simulation has been performed leading to a good agreement with experimental pictures, but missing the wavy behaviour of the instabilities occurring on the liquid sheet. Additional works are ongoing to simulate the full injector flow in one single simulation and to capture the full interaction between the internal flow and the external atomization process.

\section{Acknowledgements}

This work was granted access to the HPC resources of IDRID, TGCC and CINES under the allocation A0072B06153 and A0092B06153 made by GENCI (Grand Equipement National de Calcul Intensit) and also for the computing time at CRIANN (Centre Régional Informatique et d'Applications Numériques de Normandie) under the scientific project No. 2006011. Dr. Carreres received a "José Castillejo" aid (ref. CAS18/00289) from "Ministerio de Educación, Cultura y Deporte" in the frame of "Programa Estatal de Promoción del Talento y su Empleabilidad en I+D+i, Subprograma Estatal de Movilidad, del Plan Estatal de I+D+i". Support given to Mr. Mario Belmar by Universitat Politècnica de València through the "FPI-Subprograma 2" grant within the "Programa de Apoyo para la Investigación y Desarrollo (PAID-01-18)" is also gratefully acknowledged. Additionally, the authors would like to thank José Enrique del Rey and Ignacio Sevilla for their technical help with the silicone molding and image processing.

\section{Nomenclature}

$\begin{array}{llll}D & \text { Diameter }[\mu \mathrm{m}] & R & \text { Rounding radius }[\mu \mathrm{m}] \\ h & \text { Internal chamber height }[\mu \mathrm{m}] & S_{w} & \text { Swirl number [-] }\end{array}$


$\dot{m}_{f} \quad$ Fuel mass flow rate $\left[\mathrm{g} \mathrm{s}^{-1}\right] \quad T \quad$ Temperature $[\mathrm{K}$ ]

$p \quad$ Pressure [ MPa ]

\section{References}

[1] Lefebvre, A. H., McDonell, V. G., 2017, "Atomization and Sprays". CRC Press.

[2] Park, K. S., and Heister, S. D., 2010, International Journal of Multiphase Flow, 36, pp. 1-12.

[3] Amedorme, S. K., and Apodi, J. A., 2018, AIP Conference Proceedings, pp. 020026.

[4] Rezaei, S., Vashahi, F., Ryu, G., Lee, J., 2019, Fuel, 258, pp. 116094.

[5] Mongia, H. C., Jul. 14-17. 2003, AIAA/ICAS International Air and Space Symposium and Exposition.

[6] Leask, S. B., Li, A. K., McDonell, V. G., and Samuelsen, G. S., 2019, Journal of Fluids Engineering, 141, pp. 121407.

[7] Dafsari, R. A., Lee, H. J., Han, J., et al., 2019, Fuel, 240, pp. 179-191.

[8] Maly, M., Sapik, M., Jedelsky, J., et al., 2018, EPJ Web of Conferences, 180, pp. 02059.

[9] Liu, C., Liu, F., Yang, J., et al., 2019, Journal of the Energy Institute, 92, pp. 210-221.

[10] Sumer, B., Erkan, N., Uzol, O., Tuncer, I. H., Sept. 2-6. 2012, ICLASS 2012, 12th Triennial International Conference on Liquid Atomization and Spray Systems.

[11] Amini, G., 2016, International Journal of Multiphase Flow, 79, pp. 225-235.

[12] Alajbegovic, A., Meister, G., Greif, D., Basara, B., 2002, Experimental Thermal and Fluid Science, 26, pp. 677-681.

[13] Madsen, J., Hjertager, B. H., Solberg, T., Sept. 6-8. 2004, ILASS-Europe 2004.

[14] Laurila, E., Roenby, J., Maakala, V., et al., 2019, International Journal of Multiphase Flow, 113, pp. 371-378.

[15] Fuster, D., Bague, A., Boeck, T., et al., 2009, International Journal of Multiphase Flow, 35, pp. 550-565.

[16] Galbiati, C., Tonini, S., Weigand, B., Cossa, G. E., May 22-27. 2016, ICMF-2016 9th International Conference on Multiphase Flow.

[17] Shao, C., Luo, K., Yang, Y., Fan, J., 2017, International Journal of Multiphase Flow, 89, pp. 57-68.

[18] Workshop on Turbulent Combustion of Sprays (TCS), http://www .tcs-workshop. org/.

[19] Shum-Kivan, F., Marrero, J., Verdier, A., et al., 2017, Proceedings of the Combustion Institute, 36, pp. 2567-2575.

[20] Verdier, A., Marrero, J., Vandel, A., et al., 2017, Proceedings of the Combustion Institute, 36, pp. 2595-2602.

[21] Macian, V., Bermúdez, V., Payri, R., Gimeno, J., 2003, Experimental Techniques, 27(2), pp. 39-43.

[22] Salvador, F. J., Gimeno, J., De la Morena, J., Carreres, M., 2018, Experimental Techniques, 42(5), pp. 467-472.

[23] Smagorinsky, J., 1963, Monthly Weather Review, 91(3), pp. 99-164.

[24] Nicoud, F., and Ducros, F., 1999, Flow, Turbulence and Combustion, 62(3), pp. 183-200.

[25] Billiant, P., Chomaz, .J. M., Huerre, P., 1998, Journal of Fluid Mechanics, 376, pp. 183-219.

[26] Scardovelli, R. and Zaleski, S., 1999, Annual review of fluid mechanics, 31(1), pp. 567603.

[27] Li, J., 1995, Comptes rendus de l'Académie des sciences. Série II, Mécanique, physique, chimie, astronomie, 320(8), pp. 391-396;

[28] Rusche, H., 2002-2012.

[29] Wardle, K.E., Weller, H.G., 2013, International Journal of Chemical Engineering, 2013. 